\title{
Integrating Model-based Security Risk Management into eBusiness Systems Development
} The CORAS approach

\author{
Theo Dimitrakos* ${ }^{(a)}$, Brian Ritchie ${ }^{(a)}$, Dimitris Raptis ${ }^{(b)}$, Jan $\emptyset$ yvind Aagedal ${ }^{(c)}$, \\ Folker den Braber ${ }^{(c)}$, Ketil Stølen ${ }^{(c)}$, Siv Hilde Houmb ${ }^{(d)}$ \\ ${ }^{(a)}$ Central Laboratory of the Research Councils (CLRC-RAL), ${ }^{(b)}$ Intracom S.A, ${ }^{(c)}$ SINTEF Group, \\ ${ }^{(d)}$ Telenor S.A.
}

\begin{abstract}
Security evaluation and security assurance are important aspects of trust in ebusiness. CORAS is a European project which is developing a tool-supported framework for precise, unambiguous, and efficient risk assessment of security critical systems. The framework is obtained through adapting, refining, extending, and combining methods for risk analysis of critical systems and semiformal modelling methods. In this paper we provide an overview of the CORAS framework for model-based risk assessment, emphasising the pursued integration of risk management and semiformal modelling throughout the evolution of an iterative system development process.ut of the process.
\end{abstract}

Key words: Model based Risk Analysis, Unified Process, Modelling, Security Assessment.

\section{INTRODUCTION}

The increasing complexity of today's systems urges the improvement of existing methods of analysing systems and their specification in order to increase the likelihood that all possible threats are taken into consideration. Such an improvement can be achieved by combining different risk analysis methodologies with respect to the system architecture. For example, qualitative methodologies for analysing risk lack the ability to account for the dependencies between events, but are effective in identifying potential

- Correspondence author. CLRC Rutherford Appleton Laboratory, Oxfordshire, OX11 0QX, UK. email: t.dimitrakos@rl.ac.uk See also http://www.bitd.clrc.ac.uk/Person/T.Dimitrakos

\footnotetext{
The original version of this chapter was revised: The copyright line was incorrect. This has been corrected. The Erratum to this chapter is available at DOI: $10.1007 / 978-0-387-35617-4 \_48$ 
hazards and failures in trust within the system, whereas tree-based techniques take into consideration the dependencies between each event. We are not aware of an already developed integrated approach to system modelling and risk analysis, where the architecture expressed in the information system model is used to guide the combined application of risk analysis techniques. This need is being addressed in the European project CORAS0 for the area of security risk assessment. In this paper we provide an overview of the results obtained in the ongoing CORAS project emphasising the pursued integration of risk management and semiformal modelling throughout the evolution of an iterative system development process. We conclude by summarising the achievements of CORAS and indicating how the project results can be used in order to improve trust and confidence in information systems for e-business and e-government.

CORAS provides a tool supported framework for precise, unambiguous, and efficient risk assessment of security-critical systems. CORAS focuses on the tight integration of viewpoint-oriented UML modelling in the risk management process. In this context, CORAS emphasises the practical use of UML and UP 0et al 2002) in the context of security and risk assessment, and provides support for integrity, availability, accountability, authenticity, and reliability of IT systems.

The main output of the CORAS project is a framework for model-based risk assessment having four anchor points:

1. A risk management process based on the AS/NZS 4360 standard.

2. A risk documentation framework based on the ISO standard RM-ODP (ISO/IEC 10746 series: 1995 ).

3. An integrated risk management and development process based on UP (Jacobson et al 1999)

4. A platform for tool-inclusion based on XML (World Wide Web Consortium, 2000)

The CORAS consortium consists of three commercial companies: Intracom (Greece), Solinet (Germany) and Telenor (Norway); seven research institutes: CLRC/RAL (UK), CTI (Greece), FORTH (Greece), IFE (Norway), NCT (Norway), NR (Norway), and SINTEF (Norway); as well as one university college: Queen Mary University of London (UK). CORAS runs from January 2001 to July 2003.

\section{MODEL-BASED RISK ASSESSMENT}

We use the term "risk assessment" in order to refer to the combination of the systematic processes for risk identification and determination of their consequences, and for how to deal with these risks. Many risk assessment 
methodologies exist, focussing on different types of risks or different areas of concern. The CORAS risk assessment methodology builds on:

- HAZard and OPerability study (HAZOP) (Redmill et al 1999);

- Fault Tree Analysis (FTA) (IEC 1025: 1990);

- Failure Mode and Effect Criticality Analysis (FMECA) (Bouti et al 1994);

- Markov analysis methods (Markov) (Littlewood, 1975);

- CCTA Risk Analysis and Management Methodology (CRAMM) (Atkinson et al 2002).

These methods are to a large extent complementary. They also cover all phases in the system development and maintenance process.

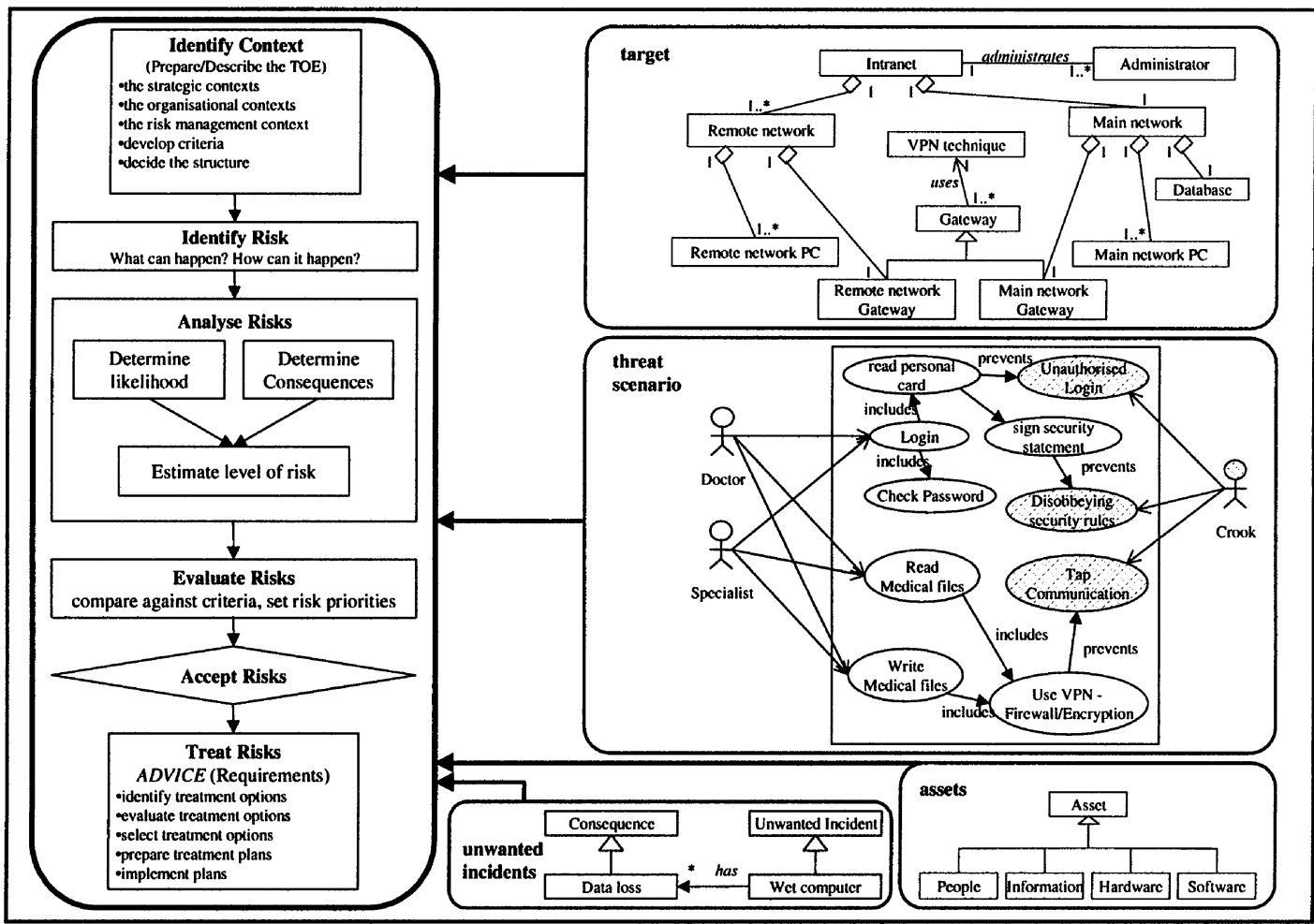

Figure 1: The role of UML in the CORAS risk management process

The CORAS risk assessment methodology incorporates a documentation framework, a number of integrated risk analysis techniques and a risk management process based upon widely accepted standards. It gives detailed recommendations for the use of UML-oriented modelling in conjunction with risk assessment.

The CORAS risk management process is based on the following standards: AS/NZS 4360:1999 "Risk Management" 0and ISO/IEC 177991:1999 "Code of Practice for Information Security Management" (ISO/IEC 17799: 2000). AS/NZS 4360 provides a sequencing of the risk management process into sub-processes for context identification, risks identification, 
risks analysis, risks evaluation, and risks treatment (as illustrated in Figure 1). The CORAS methodology provides guidelines about which models are best suited for each sub-process, and how they should be expressed. We use this process to position models within risk assessment. AS/NZS 4360 also provides two parallel processes aiming at the communication and consultation, and at the monitoring and review of each of the sequential subprocesses, hence giving rise to an iterative risk management process. The correlation between the risk management iterations and the system design and development process is elaborated in the following section.

The CORAS system documentation framework is a specialisation of the Reference Model for Open Distributed Processing (RM-ODP). Whilst incorporating RM-ODP as a whole, the CORAS system documentation framework refines only those parts of RM-ODP that are directly relevant for risk assessment of security critical systems. This is achieved by introducing two new classes of terminology - concepts for risk assessment and security and an additional structuring to the viewpoints - dividing the RM-ODP viewpoint structure into cross-viewpoint concerns targeting model-based security risk assessment.

These concerns may be understood as more specialised cross-viewpoint perspectives linking together related information within the five viewpoints. The concerns are further decomposed into models. A model provides the content of a concern with respect to a particular viewpoint. For each model there are guidelines for its development, including recommendations of which modelling languages to use. A number of identified concerns have also been related to the five sequential sub-processes of the CORAS risk assessment process (Figure 2). 


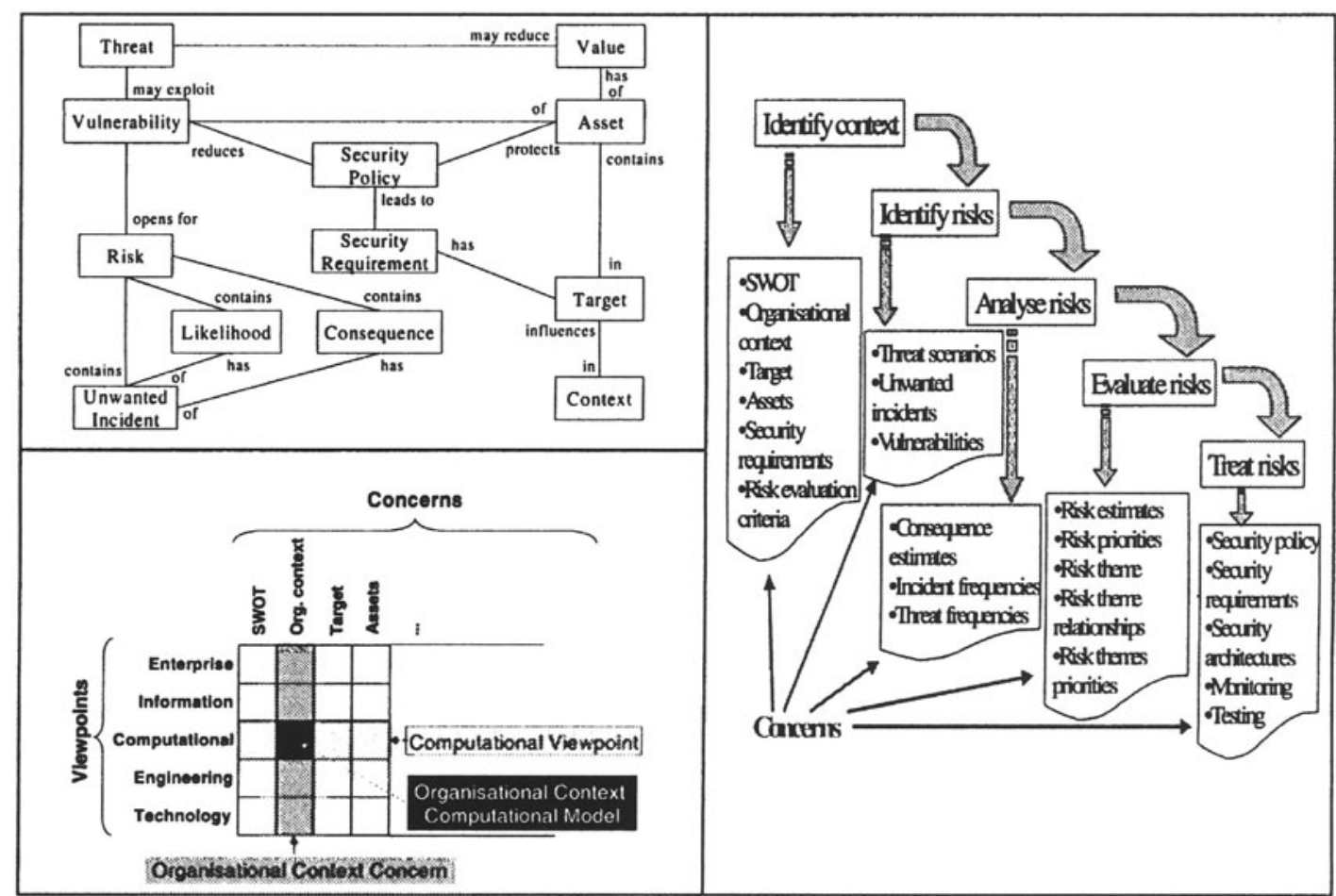

Figure 2: Anticlockwise: Basic concepts for Security Risk Assessment; Concerns, viewpoints and models; Concerns and risk management sub-processes.

Figure 1 provides an example of how modelling concepts relate to the risk management process. The four diagrams to the right of Figure 1 illustrate:

- specification of the target of evaluation with the help of a UML class diagram (aspect of the target concern depicted in Figure 2);

- specification of a threat scenario with the help of a misuse case diagram (Sindre et al, 2000) (example element of the threat scenarios concern listed in Figure 2);

- specification of the assets to be protected with the help of a UML class diagram (aspect of the assets concern listed in Figure 2);

- specification of an unwanted incident with the help of a UML class diagram (example element of the unwanted incidents concern in Figure 2).

The CORAS risk management process follows the main iterations made in the CORAS system development process, as indicated by Figure 3. Each main iteration adds more detail to the target and the context of the assessment and previous results may need to be re-evaluated. 


\section{THE INTEGRATED RISK MANAGEMENT AND SYSTEM DEVELOPMENT PROCESS}

The CORAS integrated risk management and system development process is based on an integration of the risk management process in UP supported by UML-based viewpoint-oriented modelling. In the following paragraphs we highlight the defining characteristics of the CORAS process, as summarised by Figure 3.

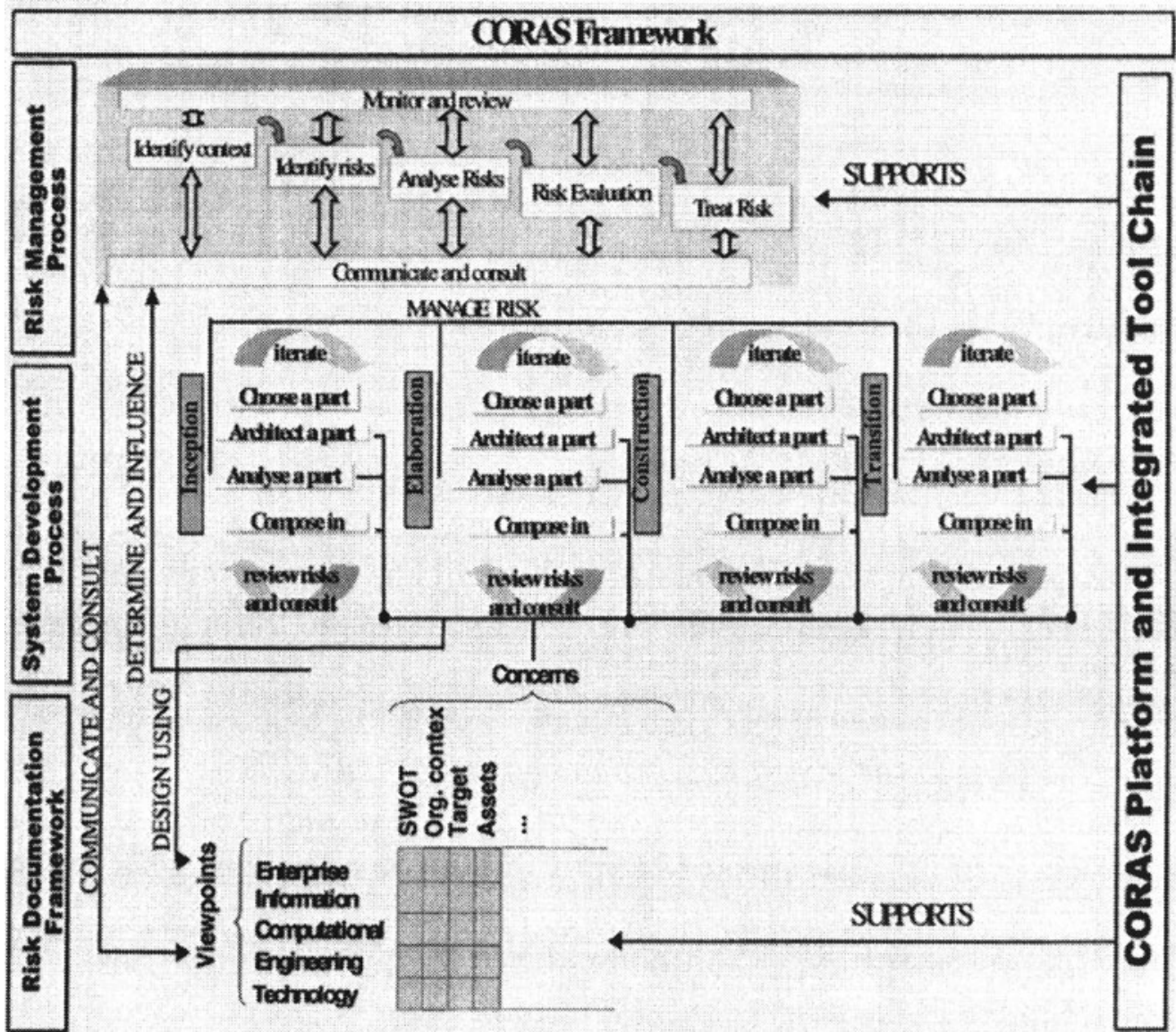

Figure 3: The integrated risk management and system development process

In the following paragraphs we highlight the defining characteristics of the CORAS process that are summarised in the visualisation seen in Figure 3.

An incremental iterative process. In analogy to RUP, the CORAS process is both stepwise incremental and iterative. In each phase of the system lifecycle, sufficiently refined versions of the system (or its model) are constructed through subsequent iterations before progressing to the next phase. In analogy to the RM-ODP viewpoints, the viewpoints of the CORAS framework are not layered; they are different abstractions of the same system focusing on different areas of concern. Therefore, information in all 
viewpoints may be relevant to all phases of the lifecycle. The CORAS risk management process follows the iterations made in the CORAS process. As more detail is added to target and the context of the analysis In each iteration, previous results may need to be re-evaluated.

Multiple risk management instances. The description of the CORAS risk management process (section 2) defines an abstract pattern that is instantiated using different risk analysis methods in order to analyse different parts of the system in different phases of the development lifecycle. The choice of method depends on the target and the context of the analysis.

Propagation of risk management instances. A single component of the system model, in one level of abstraction, may correspond to a whole subsystem, in another. ${ }^{1}$ This combination of refinement and decomposition inevitably results in the propagation of the risk analysis activity from the abstraction of the composite to its actual components.

\subsection{Interactions between risk assessment and system design}

As more information is incorporated in the context of the analysis there is always the potential that new vulnerabilities are identified (because of the choice of implementation or refinement) and this must be taken into account to the risk analysis of the composite entity. Consequently the risk management process (which includes risk analysis specific sub-processes) follows the iterations of the development lifecycle. While modelling sets the context for risk analysis and enforces revisions by refining or changing this context, security risk analysis contributes to selection of what needs to be refined and when. Security risk management guides refinement (in conjunction with cost management, deployment deadlines and implementation concerns).

The close integration between security risk management and modelling underpinning the CORAS development process gives rise to iterations of system design that are instigated by the risk assessment activities and vice versa. In addition to the usual iterations prescribed by the employed risk management and system development processes, we distinguish the following types of iterations that are due to interactions between risk management and systems design:

The iteration of the whole risk management process instigated by the change of development phase.

The iterations of the RA tasks instigated by refinement or decomposition of the system models. The behavioural or architectural structuring detail that is introduced by refinement or decomposition may allow the identification of

\footnotetext{
${ }^{1}$ This is formalised using the ODP foundation rules for abstraction and composition.
} 
new vulnerabilities or necessitate an update of the consequences of an already identified risk or lead to a revision of the risk evaluation results.

The iterations of the system design and development steps (within the same development phase) instigated by the need of the risk analyst for more information about a system aspect, which may result in a refinement or decomposition of some system models.

The iterations of the system design and development steps addressing design or implementation revisions that may be necessitated by the assessment of unacceptably high risks, due to bad design choices.

Iterations of type 2 take place for example when the description of a system component is refined to a subsystem. The RA results on the component that offers an abstract view of the subsystem both constrain the RA results of the components in the subsystem while the RA results on the later may give rise to design revisions therefore instigating a design iteration within the same development phase (iteration of type 4). Also when type 3 iteration happens, it instigates a refinement of the design at hand which may necessitate a further evaluation and perhaps making of design decisions. As such type 3 may bring about type 2 resulting in a propagation of parallel risk assessment sessions.

\subsection{Phases of the CORAS development process}

In the following paragraphs we explain the emphasis of the CORAS integrated documentation and model-based risk analysis framework from a process perspective.

Inception Phase essentials: The objective, in the initial steps, is to capture as much as possible at a sufficiently high level of abstraction leaving details to fit-in as further refinement is performed. The initial identification of the security critical components of the system to be analysed and its context takes place in the inception phase. Also the main security assets have to be identified. Outputs of this phase include abstract versions of security assessment scenarios, a list of main assets, and a definition of the system boundaries. At this phase much may be yet unknown and therefore cannot be specified. To compensate for under-specification, one needs to revisit and reevaluate what has already been specified. Both the specification and the risk analysis techniques are incremental and evolve in iterations.

Elaboration Phase: Important activities in this phase include: requirements capture, analysis, and negotiations, including several rounds with the different stakeholders, resolution of conflicting views, resolving ambiguities etc. The assessment of security requirements is informed by a combination of use and misuse scenarios. An important expected outcome of the elaboration phase is design constraints represented by a stable architectural prototype. During this phase various architectural alternatives 
are eliminated, leaving some stable selected alternatives. Risk analysis can help evaluate alternative architectures and thus inform the choice.

Construction Phase focuses on the detailed design and implementation of components that are incrementally updated. Vulnerability testing combined with quantitative risk analysis methods can be used to help with identifying, analysing and treating security critical design, implementation, and manufacturing errors. Post-implementation risk analysis combined with vulnerability testing and analysis of the implementation infrastructure may help identify and analyse risks that could not be detected in the system model. The CORAS framework supports the establishment of clear feedback channels between post-implementation analysis and system design.

Transition Phase. In addition to following up and/or finalising relevant activities from the previous phase, an important activity is the transition phase compliance testing. This involves evaluating and testing the product with respect to the vision and associated criteria as stated in the first phase of development (including security-relevant aspects). It is therefore important to involve management and system users in this phase, referring to their involvement (during the inception phase) in defining the system and its boundaries.

A set of agreed system requirements is an important outcome of the inception and elaboration phases. These requirements may be relevant to several viewpoints and can be described using a selection of different description methods, classified per concern, in accordance with Figure 2. As one cannot expect that all security requirements are present from the start, they have to be elicited. We anticipate that (appropriately adapted) modelbased security risk assessment can also help with eliciting security requirements. However, for security risk assessment to play a significant role in the elaboration phase, the CORAS risk assessment methods are being adapted to address requirement elicitation properly. For this purpose we are currently developing tailored templates to extend a scenario-driven analysis with security risk assessment throughout the development life cycle. Important sources of inspiration include the work of (Cockburn, 1997) and Schneider/Winters (Schneider et al 1998).

Another consideration with respect to improving systems security that is being addressed by the CORAS approach is to assess security policies and recommend how a system should be used in order to minimise loss or damage in the presence of identified vulnerabilities. We expect that the CORAS framework can support incorporating changes to the way the system is used or operates into the system documentation and control guidelines, targeting at the improvement of the system's security. This expectation is supported by the fact that RM-ODP allows for modelling policies and control entities in the system as well as describing policies and stakeholder roles in the enterprise context. Having established a correspondence between enterprise policies and the operational security management of the system, 
one may use model-based risk analysis, not only to assess the effectiveness of a security policy but also to decide the way in which a policy is enforced. (Policies can be enforced in a preventive, reactive or corrective manner, depending on the security risks they address weigh against implementation cost of their enforcement, the impact on the operational efficiency of the system.)

\section{THE PLATFORM FOR TOOL INTEGRATION}

The CORAS platform for tool integration is built around an internal data representation formalised in XML (World Wide Web Consortium, 2000). Based on XSLT 01999), relevant aspects of the internal data representation may be mapped to the internal data representations of other tools (and the other way around). This allows the integration of sophisticated case-tools targeting system development as well as risk assessment tools and tools for vulnerability and threat management. The CORAS platform consists of three interfaces for XML based data exchange:

- Interface based on IDMEF (Intrusion Detection Exchange Format) (Curry et al 2001). IDMEF is an XML DTD targeting tools for intrusion detection and has been developed by the Intrusion Detection Working Group.

- Interface based on XMI (XML Metadata Interchange) standardised by the Object Management Group and targeting tools for UML modelling.

- Interface targeting risk assessment tools.

The CORAS platform contains a repository divided into two parts, which follow the organisation into viewpoints and concerns outlined in Figure 2:

The assessment repository storing the concrete results from already completed assessments and assessments in progress.

The reusable modelling elements repository storing reusable models, patterns and templates from pre-defined or already completed risk assessments.

\section{A CASE STUDY}

The CORAS framework and process are being validated in extensive user trials in the areas of e-commerce and telemedicine. In this section we present the modelling approach followed in the first of the user trials (concerning the authentication mechanism of an e-commerce platform) and provides some examples of the risk analyses employed in this context. The e-commerce platform was developed in the context of the R\&D project EP-27046ACTIVE, partly funded by the European Commission under the ESPRIT programme. ACTIVE introduced a generic global e-commerce platform that 
supports integrated retail services, providing an intelligent interface upon which the involved players (retailers, suppliers and consumers) can interact. The e-commerce platform used in the CORAS trials constitutes a core part of the ACTIVE system. The user authentication mechanism of the ecommerce platform was analysed in the first CORAS trial.

\section{Context identification}

The behavioural specification of system can be expressed using UML diagrams such as State or Activity diagrams and Sequence diagrams. In particular, the overall behaviour of a Web application like the e-Commerce platform can be described as a UML state machine where each state corresponds to a specific HTML page. The state machine in Figure 4 provides a high level description of the E-Commerce platform behaviour with respect to the user authentication and identification.

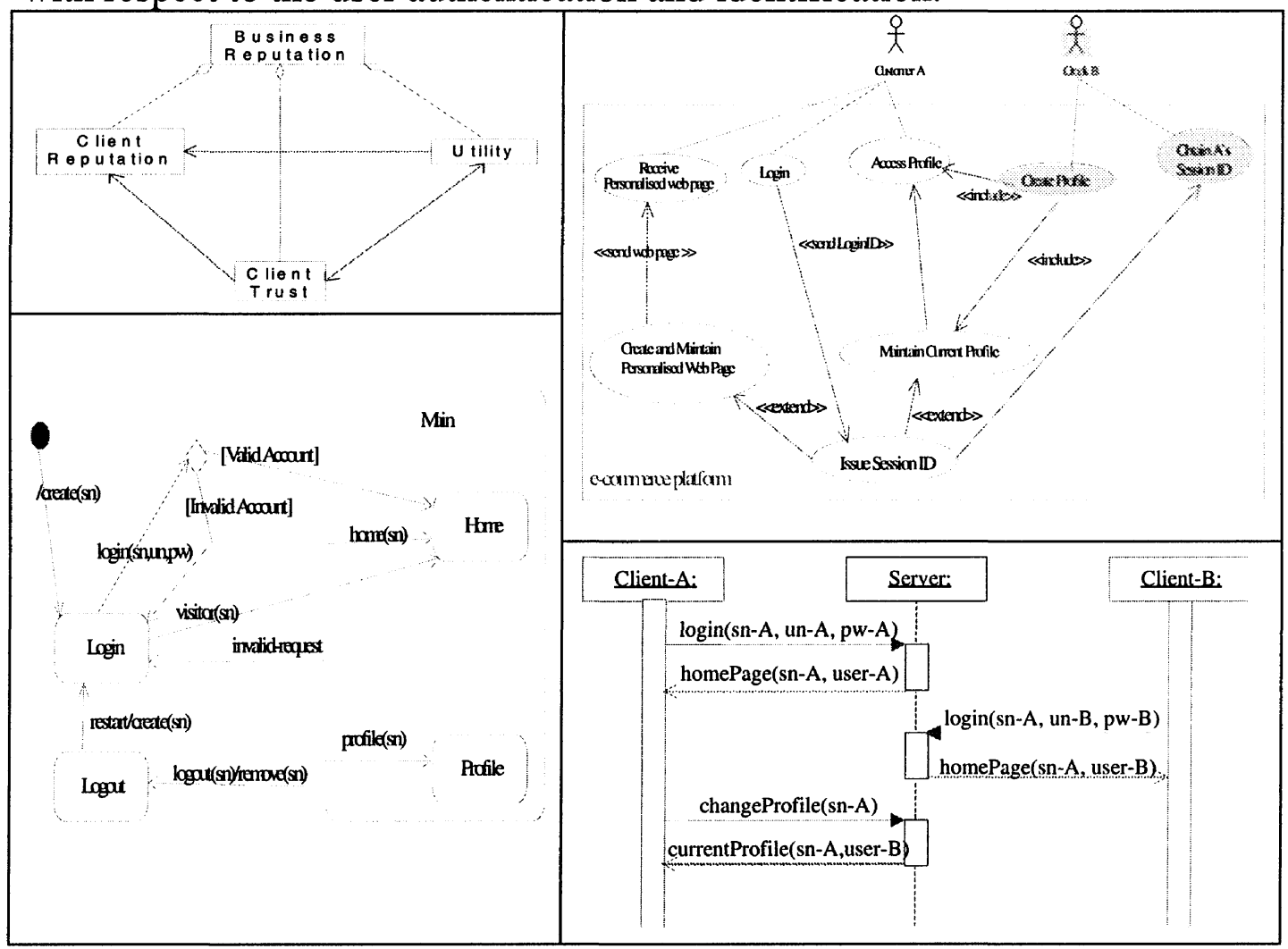

Figure 4. Anti-clockwise from top left: part of asset specification, part of user authentication behaviour, part of scenario description via a sequence diagram, part of scenario specification.

According to the diagrams in Figure 4, when a user accesses the Login page, the server creates a unique session $I D$ to identify the specific client. The session ID is used to associate each user's client with the user's data stored on the server. This session ID is sent to the user's client in all subsequent HTML pages: All HTML links contain the session ID as a parameter. In the state machine above the session ID is denoted as the parameter "(sn)". The login carries the username and password as 
parameters. Users can also access the platform as visitors without authentication but they are not able to use all functionality like shopping lists.

In addition to system behaviour and configuration, identification of assets is crucial in order to be able to perform risk assessment. For example, business reputation (of the e-Commerce platform provider) can be modelled as an aggregate asset built up from the utility of the business, the trust that its clients have in the business, the reputation of each client, etc. (Note that we look at a client as an entity having its own reputation.

\section{An illustrative scenario}

The use of session numbers for client identification can have undesirable consequences if a malicious actor captures a client's session ID. This actor can use the session ID to login in the platform using a second account with an offensive profile (e.g., vulgar names) using the legitimate user's session ID. From that point on, all interactions of the legitimate user with the platform will have the profile of the second account. An overview of this scenario (focusing on the documentation of threats and vulnerabilities) is presented by means of a misuse case diagram. The diagram includes the regular use case and a shaded use case describing the abuse of the system by a malicious actor. The corresponding behaviour of the system can be as the sequence diagram in Figure 4, which can be perceived as part of a specialisation of the scenario description presented via the misuse case:

A legitimate user, Client-A, logs in the platform with the username/password un- $\mathrm{A} / \mathrm{pw}-\mathrm{A}$, using session ID $\mathrm{sn}-\mathrm{A}$, and receives back a page personalised to Client-A user-A. After a malicious user, Client-B, logs in with the session ID of Client-A, the profile that Client-A accesses is the profile of Client-B (e.g., the Client-B's name and shopping lists).

This example is not the result of implementation defects, but a consequence of the design decision to use session IDs for client identification. Notably the use of cookies is subject to similar deficiencies (Fu et al 2001).

\section{Risks assessment}

In order to identify risks, risk analysis is performed. The risk analysis of the user authentication mechanism deployed by the e-commerce platform was based on models of its behaviour like those presented in the previous subsection. Initially CRAMM was applied in order to provide an identification of assets, which in turn provide a basis and justification for the security requirements that the mechanism need to meet. Then HazOp, FMEA and FTA were performed, and some examples of this are presented below. 
The objective of HazOp is to identify possible unwanted incidents, as well as their causes and consequences. Starting with the system's behaviour as expressed by the state machine in Figure 4, all events are independently analysed. As an example, an excerpt of HazOp applied on a user's request to access the Login page ("^ create(sn)" event) is presented in Figure 5. The first column, Entity, corresponds to the events of the system behaviour followed by a brief informal description. The Security attributes correspond to possible breach of security requirements of confidentiality, integrity availability and accountability. The deviations column presents deviations from normal or expected behaviour, like undesirable (accidental or malicious) interactions with the system. The next columns presents possible causes that enable or cause the deviations, and the consequences of these deviations. The Actions column presents some steps that can be taken to avoid or mitigate the risk of the deviation to occur. Some Remarks are presented in the last column.

FMEA was used to identify possible failure modes of individual components. For software systems, like the e-commerce platform, these failures can be wrong results and exceptions or error values returned by function calls to software components. Due the large size of modern software systems, the FMEA table becomes rapidly very large and time consuming to produce. The CORAS trials therefore focused only on small parts of the Web, Application and Database servers of the e-commerce platform. The objective of Fault Tree Analysis is to document in a structured way the possible routes that can lead to the violations of security requirements identified by $\mathrm{HazOp}$ or failures identified by FMEA. As an example, an excerpt of a Fault Tree demonstrating some possible routes that lead to breach confidentiality by accessing a user's personal data in the ecommerce platform is presented in Figure 5. The nodes of a fault tree are called event blocks, and the root node called top-event. The OR-gates join alternative means that can lead to their parent nodes. The round circles indicate that the parent events are basic events that are not analysed further. In this example, the tree is a branch of larger tree that covers all range of violations of security requirements. There are also more situations resulting in state S0, like circumventing the web server or internal fraud, but these are not presented here.

There is a close relation between the deviations identified by HazOp analysis, the possible failures identified by FMEA and the fault tree constructed in that these deviations appear as nodes ("event blocks") in the fault tree. For example, item 1.1.2 of HazOp table identified that a capture of a server response leads to the disclosure of Session ID. This is reflected in FTA tree, where state S4 can be achieved by means of reaching state S7. When the risks are identified, analysis of impact and likelihood are performed. The results of this analysis typically annotate existing models or one creates tables listing the risks and assigning likelihood and consequences 
to them. Similarly, the risk evaluation activity produces levels of risk and risk priorities that further annotate existing specifications.

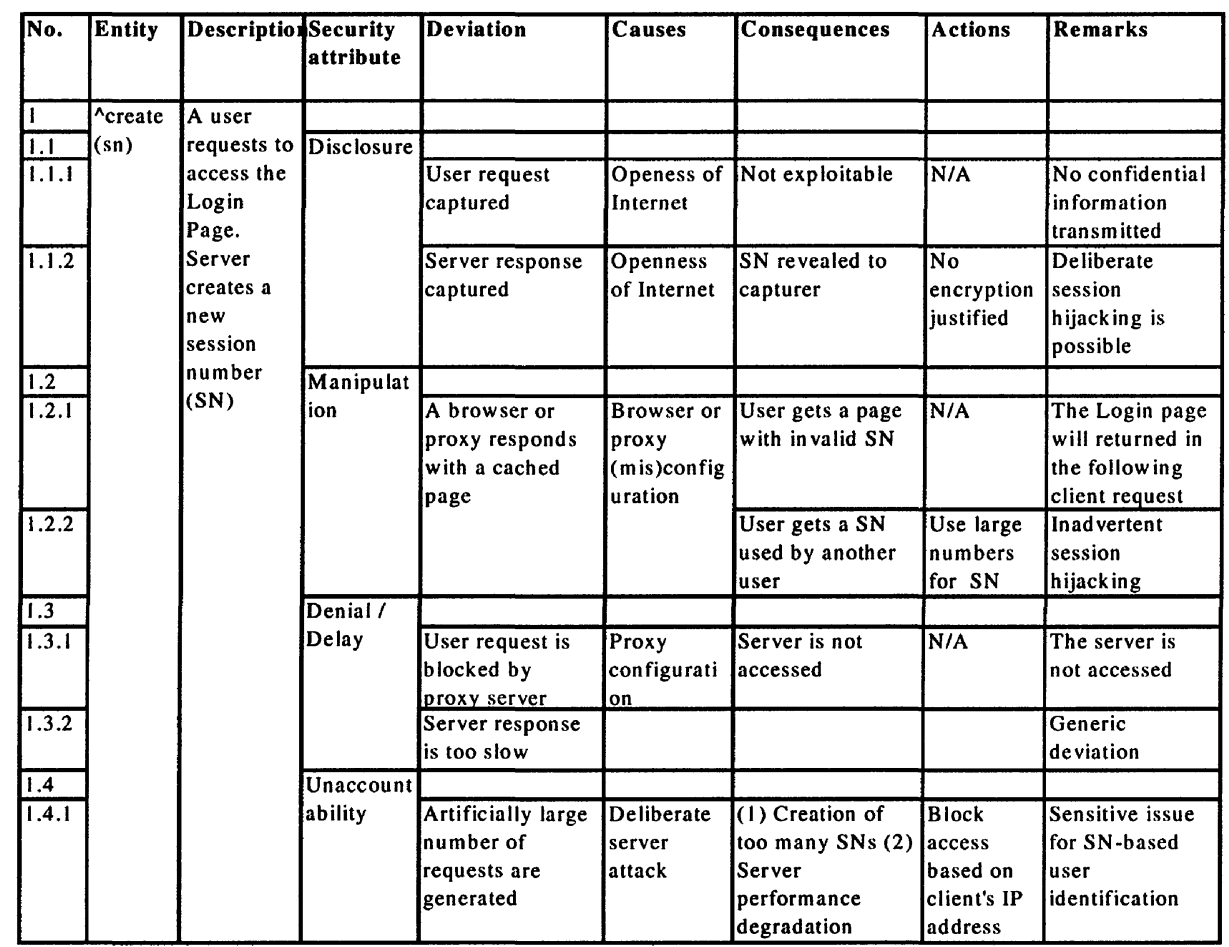

Figure 5: HazOp table for the Login page.

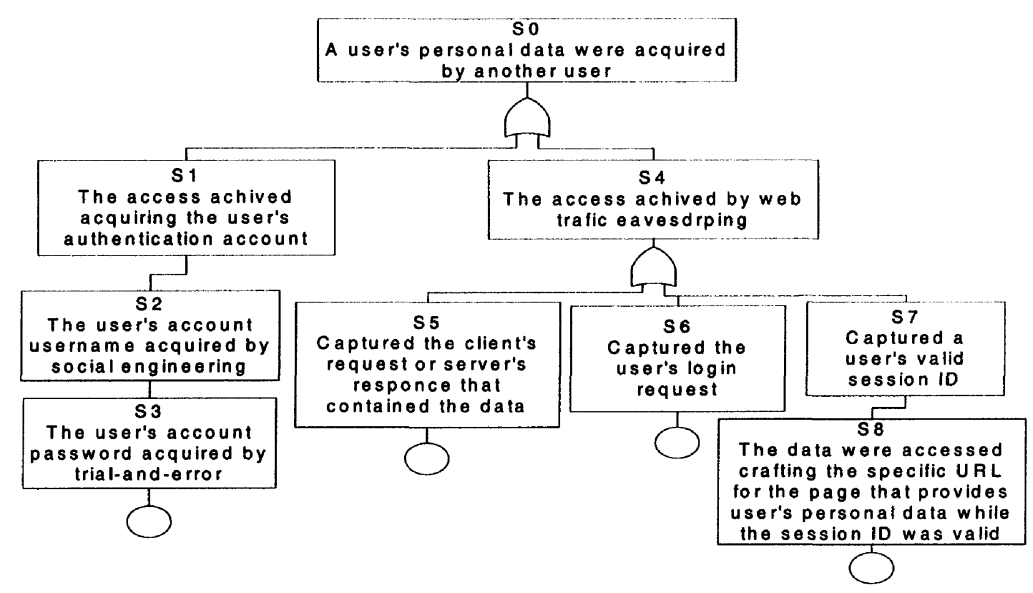

Figure 6: Fault tree example related to the HazOp table of Figure 5.

\section{RELATED WORK}

Since 1990, work has been going on to align and develop existing national and international schemes in one, mutually accepted framework for testing IT security functionality. The Common Criteria 0 (CC) represents the outcome of this work. Increasingly it is replacing national and regional 
criteria with a worldwide set accepted by the International Standards Organisation (ISO15408) (ISO/IEC TR 13335-1:2001). The CC and CORAS are orthogonal approaches. The $\mathrm{CC}$ provides a common set of requirements for the security functions of IT products and systems, and a common set of requirements for assurance measures applied to the IT functions of IT products and systems during a security evaluation. CORAS provides CORAS addresses and develops concrete specification technology specifically addressing risk analysis.

Surety Analysis (SA) (Sandia National Laboratories, 2002), is a methodology based on the creation of an explicit model that covers several aspects of the system's behaviour. OPRA, the tool that has been implemented to support SA, has a "tight" integration of existing commercial software packages with tools developed in Sandia Labs especially for this project. The CORAS platform will facilitate a "loose" integration platform based on widely deployed interchange standards that will allow for different users to adapt the CORAS platform to their own needs.

RSDS is a tool-supported methodology developed by King's College London and B-Core UK, ltd. CORAS focuses on security risk analysis whereas current work on RSDS focuses on safety and reliability analysis.

The Control Objectives for Information and related Technology (COBIT)0 focuses on control objectives defined in a process-oriented manner following the principles of business re-engineering. COBIT is therefore orthogonal to CORAS.

CCTA Risk Analysis and Management Methodology (CRAMM) 0et al 1992) was developed by the British Government's Central Computer and Telecommunications Agency (CCTA) with the aim of providing a structured and consistent approach to computer security management for all systems. The UK National Health Service considers CRAMM to be the standard for the risk analysis of information systems within healthcare establishments. CRAMM is an important source of inspiration for CORAS, and aspects of CRAMM have been incorporated in CORAS. Contrary to CRAMM, CORAS provides a risk analysis process in which modelling is tightly integrated, and CORAS complies with state-of-the-art international standards for risk management, documentation, modelling and development of systems. CCTA has extended CRAMM into an overall system development process by developing an interface between CRAMM and SSADM (Structured Systems Analysis and Design Method). This corresponds to the CORAS integrated risk management and system development process based on AS/NZS 4360 and UP. 


\section{CONCLUSION}

Information and communication technologies (ICT) are becoming a dominant part of people's everyday life in Europe. The demand for trusted and cost-effective security solutions is increasing as the use of decentralised distributed systems built on open networks proliferates. Such systems are in many cases vital for the enterprise-wide functions. The CORAS approach provides one way of incrasing trust and confidence in information and communication systems based on the integration of security risk management and graphical semiformal modelling for the efficient and unambiguous security assessment of such systems. In particular we anticipate that the integration of risk analysis and semiformal modelling, which underpins the CORAS framework, will provide several benefits, including:

- An improvement to the current state-of-the-art of methods for risk analysis;

- The ability to handle more complex systems, analysed at different levels of abstraction;

- The ability to adapt the same framework for analysing legacy systems and systems under development, depending on the user needs;

- The ability to re-use a part of the risk analysis results during system maintenance, for example when new subsystems or components are introduced in an already analysed system,;

- The ability to use the system model architecture in order to guide the couse of complementary risk analysis methods in a way that increases the effectiveness of the overall result.

Future extensions of the work undertaken within CORAS may involve extending the integrated risk analysis and systems modelling framework of CORAS with a model of trust (such as the model outlined in (Dimitrakos, 2001) and elaborated in (Dimitrakos et al 2001) in order to support analysing trust in e-services.

\section{REFERENCES}

Australian/New Zealand Standard AS/NZS 4360:1999: Risk Management.

Atkinson, C., Bayer, J., Bunse, C., Kamsties, E., Laitenberger, O., Laqua, R., Muthig, D., Paech, B. Wüst, J., Zettel, J., Component-based product line engineering with UML. Addison-Wesley, 2002.

Barber, B., Davey, J., The use of the CCTA risk analysis and management methodology CRAMM. Proc. MEDINFO92, North Holland, 1589 -1593, 1992.

den Braber, F., Dimitrakos, T., Gran, B.A., Stølen K., Aagedal, J.Ø. Model-based Risk Management using UML and RUP, Issues and Trends of Information Technology Management in Contemporary Organizations 2002, Information Resources Management Association International Conference, May 2002. 
Bouti, A., Ait Kadi, D. A state-of-the-art review of FMEA/FMECA. International Journal of Reliability, Quality and Safety Engineering 1:515-543, 1994.

Clark, J., XSL transformations (XSLT) 1.0, World Wide Web Consortium recommendation REC-xslt, November 1999.

Cockburn, A., Structuring use cases with goals. Journal of object-oriented programming, Sep/Oct: 35-40, Nov/Dec: 56-62, 1997.

Common Criteria Organisation, “Common Criteria for Information Technology Security Evaluation", http://www.commoncriteria.org, accessed: 2002.

Control Objectives for Information and related Technology, "COBIT", http://www.isaca.org/ct_denld.htm

CORAS project web-site. http://www.nr.no/coras

Curry, D., Debar Merrill Lynch, H. Intrusion detection message exchange format (IDMEF). Working draft, December 28, 2001.

Dimitrakos Th., Bicarregui J.C. " Towards A Framework for Managing Trust in e-Services". In Proceedings of the $4^{\text {th }}$ International Conference on Electronic Commerce Research, ATSMA, IFIP, November 2001. ISBN 0-9716253-0-1.

Dimitrakos Th. "System Models, e-Risk and e-Trust. Towards bridging the gap?" in Towards the E-Society: E-Business, E-Commerce, and E-Government, eds. Schmid B., StanoevskaSlabeva K., Tschammer V., Kluwer Academic Publishers, 2001. ISBN-0-7923-75297

K. Fu, E. Sit, K. Smith and N. Feamster, Dos and Don't of Client Authentication on the Web, MIT Technical Report 818, MIT Laboratory for Computer Science, 2001. http://cookies.lcs.mit.edu/webauth:tr.pdf

ISO/IEC 10746 series: 1995 Basic reference model for open distributed processing.

ISO/IEC TR 13335-1:2001: Information technology - Guidelines for the management of IT Security-Part 1: Concepts and models for IT Security.

ISO/IEC 17799: 2000 Information technology - Code of practise for information security management.

IEC 1025: 1990 Fault tree analysis (FTA).

Jacobson, I., Rumbaugh, J., Booch, G. The unified software development process. AddisonWesley, 1999.

Littlewood, B. A reliability model for systems with Markov structure. Appl. Stat. 24:172-177, 1975.

Reactive System Design Support, “RSDS”, http://www.dcs.kcl.ac.uk.

Redmill, F., Chudleigh, M., Catmur, J. Hazop and Software Hazop. Wiley, 1999.

Sandia National Laboratories, "Surety Analysis", http://www.sandia.gov, 2002.

Schneider, G., Winters, J. P. Applying use cases: a practical guide. Addison-Wesley, 1998.

Sindre, G., Opdahl, A. L. Eliciting security requirements by misuse cases. In Proc. TOOLS_PACIFIC 2000. IEEE Computer Society Press, 120-131, 2000.

World Wide Web Consortium, Extensible Markup Language (XML) v1.0, W3C Recommendation, Second Edition, 6 Oct. 2000. 